\title{
Characterisation of borosilicate glass media as potential thermoluminescent dosimeters
}

\author{
Amal Alqahtani ${ }^{1,2^{*}}$, D. A. Bradley ${ }^{1,3}$, Abdulaziz Alanizi ${ }^{1,4}$, Andrew Nisbet ${ }^{5}$ \\ ${ }^{1}$ Department of Physics, University of Surrey, Guildford GU2 7XH, United Kingdom \\ ${ }^{2}$ College of Medicine, University of Imam Abdulrahman Bin Faisal, Dammam, Saudi Arabia \\ ${ }^{3}$ Sunway University, Centre for Biomedical Physics, Jalan Universiti 47500 Subang Jaya, Malaysia. \\ ${ }^{4}$ Medical Physics Department, Cancer Centre, Prince Mohammed Medical City, Aljouf, Saudi Arabia \\ ${ }^{5}$ Department of Medical Physics \& Biomedical Engineering, University College London, London WC1E 6BT, UK
}

*Corresponding author: a.m.alqahtani@surrey.ac.uk

\begin{abstract}
:
Thermoluminescence dosimetry applications range from environmental radiation monitoring to sensing of the doses delivered in radiotherapy, through to verifying the doses of large-scale industrial irradiation. Particular utilisations largely depend upon the sensitivity of the dosimetric medium. In present work the thermoluminescent dosimetric properties of commercial low-cost borosilicate glass slides and glass-fibre filters were characterized, use being made initially of a $250 \mathrm{kV}$ clinical therapy x-beam facility. From this, reproducibility and linearity were determined for radiation doses of $0.5,1,2,4,6,8$, and $10 \mathrm{~Gy}$. Reproducibility of $+15 \%$ was obtained following appropriate screening, excellent linearity to dose also being demonstrated for both the borosilicate glass slides and glass-fibre filters, sufficient to demonstrate the potential of these media for use in radiotherapy dosimetry. Of note is the appreciable but not unsurprising dose response of the nominal $1.0 \mathrm{~mm}$ thickness borosilicate glass slides, with a per unit dose response some twice that of the less substantial glass-fibre filters. Further work has characterized the borosilicate glass slides for the megavoltage photons and electrons produced by linacs. A further potential interest results from the boron content, recognizing the associated appreciable neutron cross-sections. It is posited that the additional response observed at $10 \mathrm{MV}$ over that at $6 \mathrm{MV}$ is partly a result of photo-neutron production, a matter that is expected to form the basis of further investigations.
\end{abstract}

Keywords: borosilicate glass; thermoluminescence, dosimetry, radiotherapy

\section{Introduction:}

Thermoluminescence refers to the literal emission of light upon heating of an insulating or semiconducting sample subsequent to its irradiation. For dose measurements, ideally the quantity of light emitted should be proportional to the absorbed dose within the dosimetric medium. Given this and adequate response, the TL technique can be applied to environmental and personnel levels through to high-dose dosimetry, doses ranging from the sub mGy through to many tens of $\mathrm{kGy}$, the latter activities including aspects of gammagraphy, sterilization of surgical materials, food irradiation, polymerization of cables and nuclear activities (Knoll, 2000).

To-date, the thermoluminescent (TL) materials used for the many such applications have generally been ionic crystals, the phenomenon of TL being described in terms of the energy band model; for crystal structures doped by impurities, capture centres (traps) may be created in the forbidden band. The TL signal shows peaks at temperatures that are associated with release from the electron-hole traps. Of note is that the many emergent TLcharacteristics of non-conductive amorphous media, all familiar in use of crystalline media, can also find effective interpretation through adoption of the energy band model (Bradley et al., 2015, 2016).

For effective TL dosimetry important features include high sensitivity, linear response for a wide dose range, stability and a simple glow curve (Laboratory Resource, 2019). A range of materials, natural and artificial, have 
found popularity in TL studies, examples of the former being $\mathrm{BeO}, \mathrm{Al}_{2} \mathrm{O}_{3}$ and $\mathrm{CaF}_{2}$ (fluorite), while among the artificial materials are: $\mathrm{LiF}: \mathrm{Mg}, \mathrm{Ti}$; $\mathrm{LiF}: \mathrm{Mg}, \mathrm{P}, \mathrm{Cu}$; $\mathrm{LiF}: \mathrm{Dy} ; \mathrm{CaSO}_{4}: \mathrm{Dy} ; \mathrm{Li}_{2} \mathrm{~B}_{4} \mathrm{O}_{7}: \mathrm{Mn} ; \mathrm{Li}_{2} \mathrm{~B}_{4} \mathrm{O}_{7}: \mathrm{Cu}$, the most popular in dosimetry tending to be $\mathrm{LiF}: \mathrm{Mg}, \mathrm{Ti}$, in the commercial form referred to as TLD-100 (Laboratory Resource, 2019). Dosimetric research has tended to focus on improving the characteristics of TL materials, either through their preparation using different methods of synthesis and/or doping with different impurities (Allahverdi et al., 1999; IPEM Report 81, 1999). One of the advantages of using glass as a radiation detector is that it can be produced in small size, as in fibre form, providing utility in terms of spatial resolution, significantly so when compared against the regular form disc and chip TL phosphors such as TLD-100 (the chips typically of some few mm dimensions), while another advantage is low-cost (Turner, 2008), in addition to widespread availability.

Present study concerns the possible dosimetric utilization of commercial borosilicate glass (in the form of glass microscope slides and microfibre filters, of nominal $1 \mathrm{~mm}$ glass and sub-mm thickness respectively) at fractionated radiotherapy levels. Such media have indicative elemental composition as detailed in presently measured values, recorded in Table 1 below, comparison being made with National Institute of Standards and Technology (NIST) values. In particular, dosimetric utility was characterized for both the glass slides and microfibre filters using the TL technique, doses being delivered using kilovoltage and megavoltage x-rays and $\mathrm{MeV}$ electrons. For comparison of response in use of TL phosphors in radiotherapy dosimetry, see for instance Yaakob et al. (2011) and Liuzzi et al. (2015), TLD-100 being utilized in both cases, the former comparing against silica fibres for 6 and $10 \mathrm{MV}$ photon beams. In the latter case, regarding intraoperative electron therapeutic application for doses from sub-Gy to $10 \mathrm{~Gy}$ as herein, availability of thin dosimeters are of particular importance.

\section{Materials and Methods:}

\section{Preparation of samples:}

Present work involves three types of amorphous glass sample. The first is based on the use of commercial lab glass microscope slides (sometimes also referred to as cover glass), typically of areal dimensions 76.2 x $25.4 \mathrm{~mm}^{2}$ and with thickness in the range 1.0-1.2 mm (although slides of thicknesses of a few $\mathrm{mm}$ are also available). Cutting down to a size favourable for a typical TL reader was undertaken using a Struers Accutom-5 cutting machine. Commonly referred to as borosilicate glass, the slides are rich not only in terms of doping, including boron, but also defects, making it perhaps one of the cheapest of all manufactured glass. The boron adds interest in terms of neutron detection capability, boron having a high thermal neutron capture cross-section, a matter that is intended to be the subject of more detailed future investigations.

The second sample type investigated were borosilicate glass-microfibre filters, again similar in composition to Pyrex ${ }^{\circledR}$, acknowledging elemental concentrations to vary, presumably with adjustments made to provide for the needs of typical applications. As previously referred to, The National Institute of Standards and Technology (NIST) has provided data on the composition of typical Pyrex® glass (Table 1) (Physics.nist.gov, 2019). Also included are present measured compositional analyses of the glass slides using wavelength-dispersive $\mathrm{x}$-ray spectroscopy (WDS), enabling account of the presence of very low atomic number elements eg boron. It is to be stressed that variations between the formulations used by different manufacturers of Pyrex are to be expected, as previously noted; accordingly, the differences seen between the NIST values and present sample values are of no surprise. 
Table 1. Compositional analysis of typical Pyrex ${ }^{\circledR}$ (Physics.nist.gov, 2019) compared against that of the particular borosilicate glass-slides studied herein, use being made of wavelength-dispersive x-ray spectroscopy (WDS).

\begin{tabular}{|l|l|l|l|}
\hline \multirow{2}{*}{ Element } & \multirow{2}{*}{$\begin{array}{l}\text { Atomic } \\
\text { Number }\end{array}$} & Weight fraction $(\%)$ \\
\cline { 3 - 4 } & & NIST & $\begin{array}{l}\text { Present } \\
\text { Measurement }\end{array}$ \\
\hline $\mathrm{B}$ & 5 & 4.01 & 3.85 \\
\hline $\mathrm{C}$ & 6 & - & 1.30 \\
\hline $\mathrm{O}$ & 8 & 54.00 & 43.50 \\
\hline $\mathrm{Na}$ & 11 & 2.82 & 9.40 \\
\hline $\mathrm{Al}$ & 13 & 1.16 & 0.80 \\
\hline $\mathrm{Si}$ & 14 & 37.72 & 33.80 \\
\hline $\mathrm{K}$ & 19 & 0.33 & 0.40 \\
\hline $\mathrm{Ca}$ & 20 & - & 4.80 \\
\hline $\mathrm{Mg}$ & & - & 2.30 \\
\hline
\end{tabular}

The disc shaped glass fibre filters are purposed for high-efficiency lab filtration and are typically of low sub-mm thickness (but can even be less), also being available in pore sizes from low $\mu \mathrm{m}$ values through to the several tens of $\mu \mathrm{m}$ range, the small thickness values making them particularly interesting for dosimetry of the high dose gradients observed in the use of $\mathrm{MeV}$ electrons. For present investigations use was made of $0.26 \mathrm{~mm}$ thick glass fibre filters, a standard hole punch being used to make samples of diameter $6 \pm 0.5 \mathrm{~mm}$. The low cost and ease of sample production in use of this medium is to be stressed.

The third of the sample types investigated were Fused Silica Wafers, otherwise known as Fused Quartz Wafers, the $\mathrm{SiO}_{2}$ being in the amorphous phase. In contrast to the borosilicate glass, fused silica has no additives, with an expected low TL response, the lack of defects allowing a TL baseline against which the other two media can be judged. These have been prepared in chip form of dimension $5 \times 5 \mathrm{~mm}^{2}$. During irradiation, the three types of samples were placed over a tissue equivalent material in order to obtain standardised results, allowing for in vivo comparisons to be made (Allahverdi et al., 1999). All three glass media enjoy a high melting point, making them suitable for interrogation using a standard TLD reader operating within the typical temperature range.

\section{Readout and Glow curves:}

The basic operational components of the Riso TL/OSL reader (model TL/OSL-DA-20) utilised herein are shown in Fig. 1, being: the light detection system, luminescence stimulation system (thermal and optical) and irradiation source. Fig. 2 shows the set-up in its entirety. The light detection system comprises of a photomultiplier tube (PMT) in combination with suitable detection filters while the luminescence stimulation system comprises of a heating element and an optical stimulation unit. The measurements are all carried out in a vacuum chamber. 


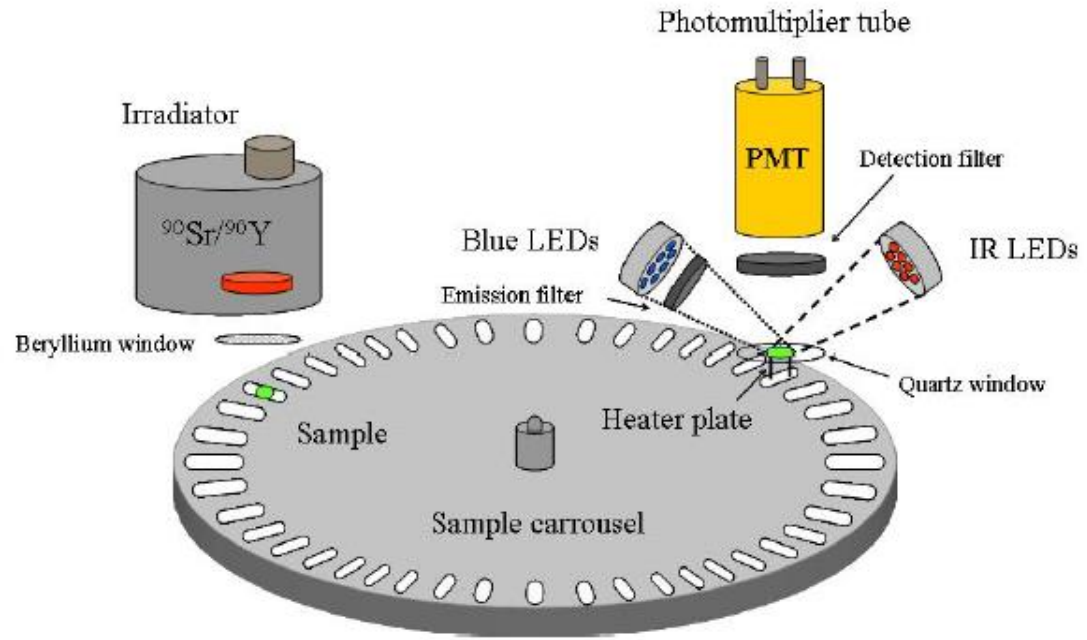

Figure 1. Schematic of the Riso combined irradiator and readout system

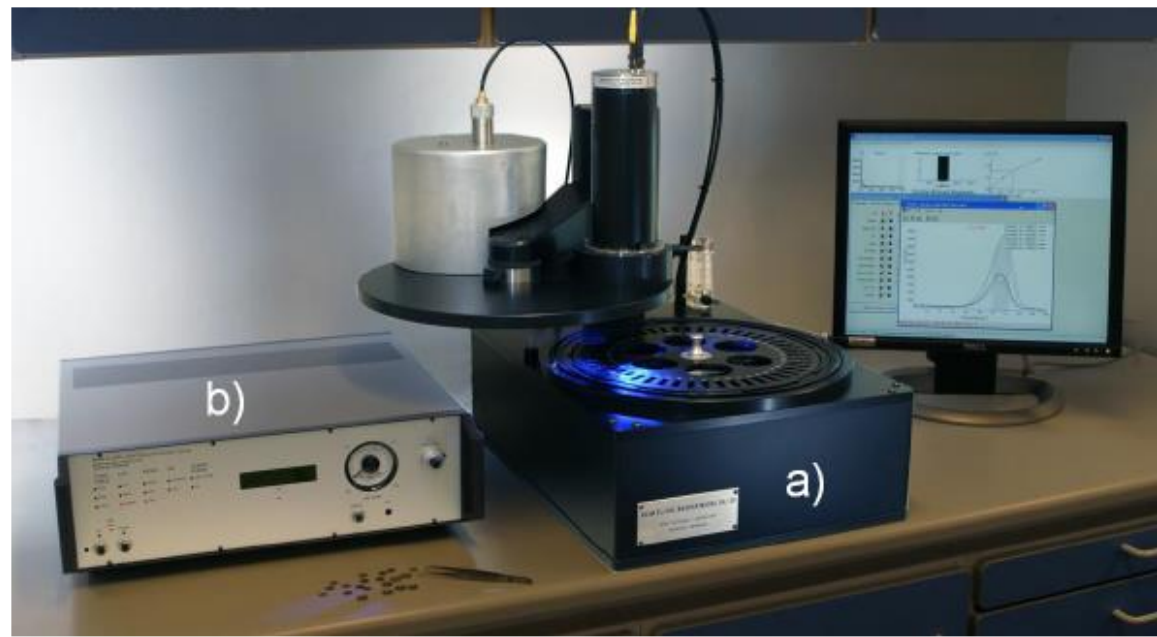

Figure 2. The Riso combined irradiator and readout system

A readout temperature of $370^{\circ} \mathrm{C}$ for 34.5 seconds was selected as suggested by others for silica glass media (Ley et al, 2019), obtained at a heating rate cycle of $10^{\circ} \mathrm{C} \mathrm{s}^{-1}$ and holding time of 20 seconds, making for a total time of readout of each sample of 54.5 seconds. With the weight of each sample dosimeter having been measured, the TL yield per unit mass was obtained, quoted in the results herein.

\section{Irradiation procedure:}

All TLD irradiations were carried out at the Royal Surrey County Hospital NHS Foundation Trust, use first being made of a Gulmay medical superficial and orthovoltage x-ray unit (Fig 3a) operating at a potential of $250 \mathrm{kVp}$, with doses ranging from 0.5 Gy to $10 \mathrm{~Gy}$ being investigated. A $20 \times 20 \mathrm{~cm}^{2}$ applicator was employed. For highenergy measurements of photons and electrons, a Varian Truebeam linear accelerator was employed (Fig 3b), investigating doses ranging from $0.10 \mathrm{~Gy}$ to $20 \mathrm{~Gy}$. For each dose, simultaneous exposure was made of all three types of glass media (glass slides, glass microfibres and fused silica wafer chips). 


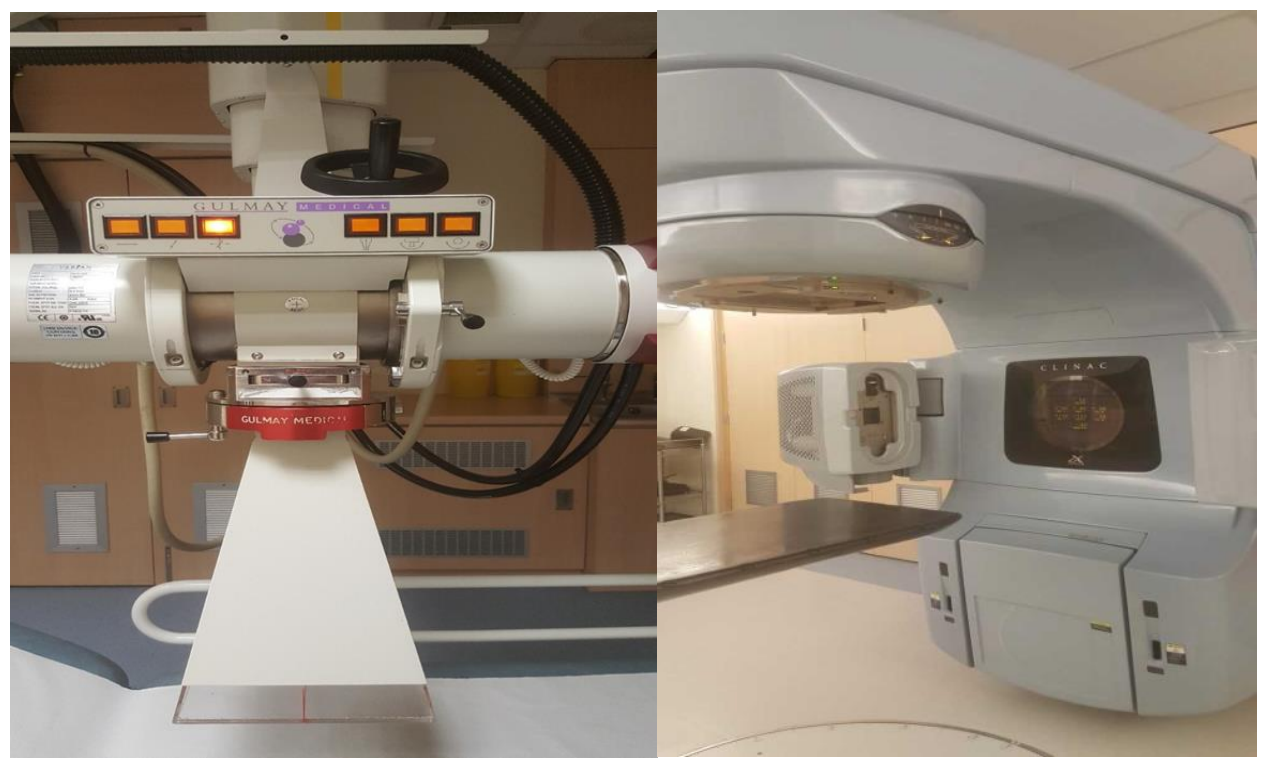

(a)

(b)

Figure 3 The two irradiation facilities used herein: (a) Gulmay medical superficial and orthovoltage $x$-ray unit, seen here with a large field size $(20 \times 20 \mathrm{~cm})$ applicator; (b) Varian Truebeam linear accelerator.

\section{Results and Discussion:}

1. Linearity of dosimeters investigated using $250 \mathrm{kVp} x$-rays:

In respect of both the glass slides and microfibre filters, the TL yield per unit mass normalised to that at $1 \mathrm{~Gy}$ shows linearity of response from $0.5 \mathrm{~Gy}$ through to $6 \mathrm{~Gy}$ (Fig 4), the irradiations being made at $250 \mathrm{kVp}$. The error bars are represented as the standard deviation arising from analysis of repeat measurements. Of note is the appreciable but not unsurprising dose response of the nominal $1.0 \mathrm{~mm}$ thickness borosilicate glass slides, with a per unit dose response some twice that of the less substantial glass-fibre filters, the major influence expected to be the greater energy absorption obtained in the rathermore dense glass network of the former, other influences not withstanding. The dosimetric potential arising from the sensitivity of the microfilters is a matter of note, not least with expected utility for electron therapy dosimetry as well as for high-dose $\mathrm{x}$-ray radio-diagnosis techniques such as angiography and CT examinations. For the silicon wafers a null response was found as expected (Fig. 5), the low defect density of such media being taken into account (TL depending upon the existence of trapping centres). Fig. 6 provides an example glow curve of borosilicate glass slides irradiated to a dose of $10 \mathrm{~Gy}$ at $250 \mathrm{kVp}$. The simple glow curve peaks at some $360{ }^{\circ} \mathrm{C}$, the minimal contribution to TL yield at temperatures below $100{ }^{\circ} \mathrm{C}$ also being noted, fading therefore being expected to be a minor influence, as currently under study. 


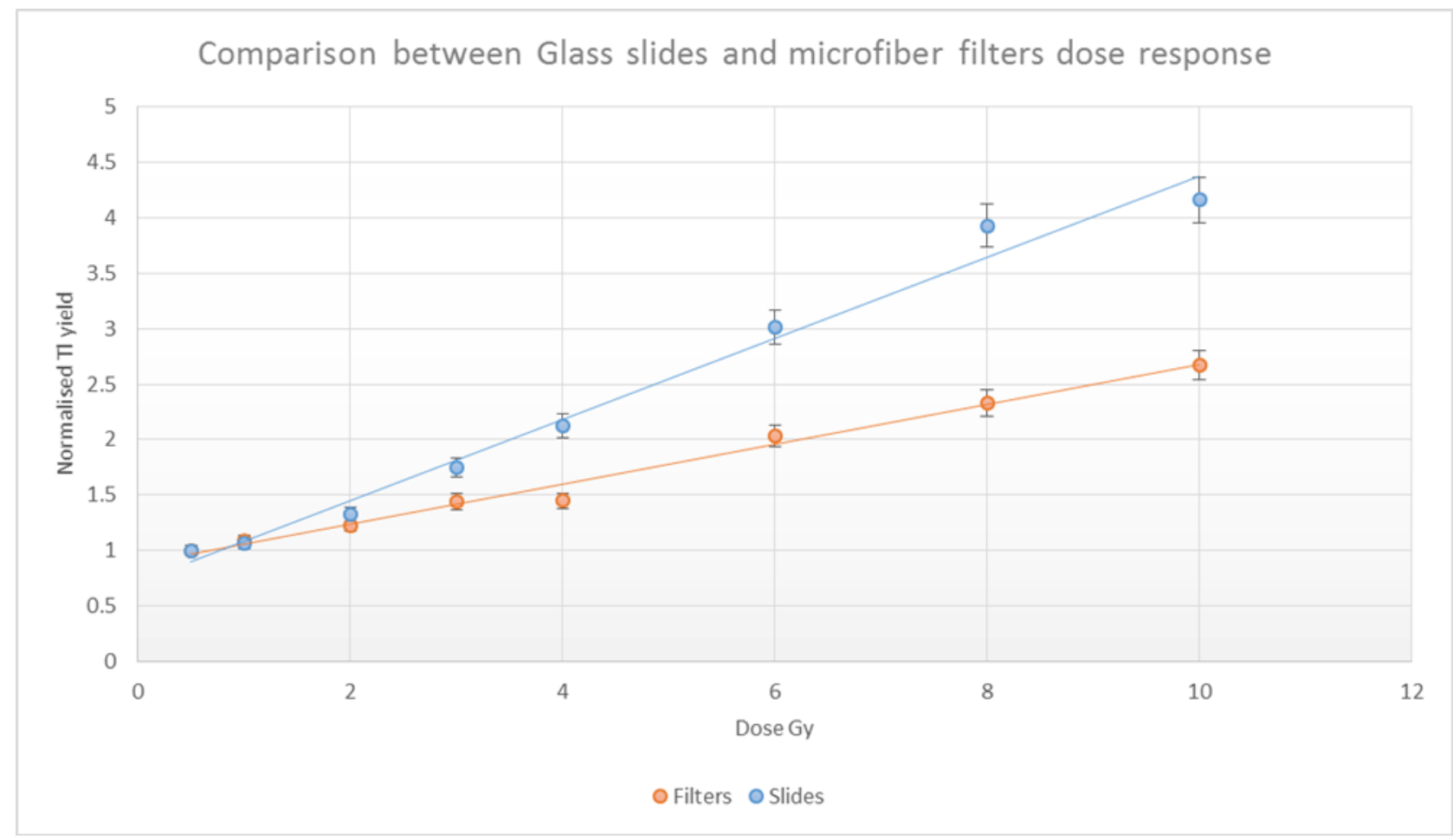

Figure 4. Readout of the glass slide (the greater slope data) and microfibre samples irradiated at eight dose points, each dose point being represented as the normalised mean TL yield per unit mass of a group of five samples in the case of the glass slides and four samples in the case of the microfibre filters. Apparent is linearity over the therapeutically useful range of doses, from below 1 Gy through to 10 Gy. The lower slope-data represents the results for the glass microfibre samples, again irradiated using the Gulmay x-ray unit.

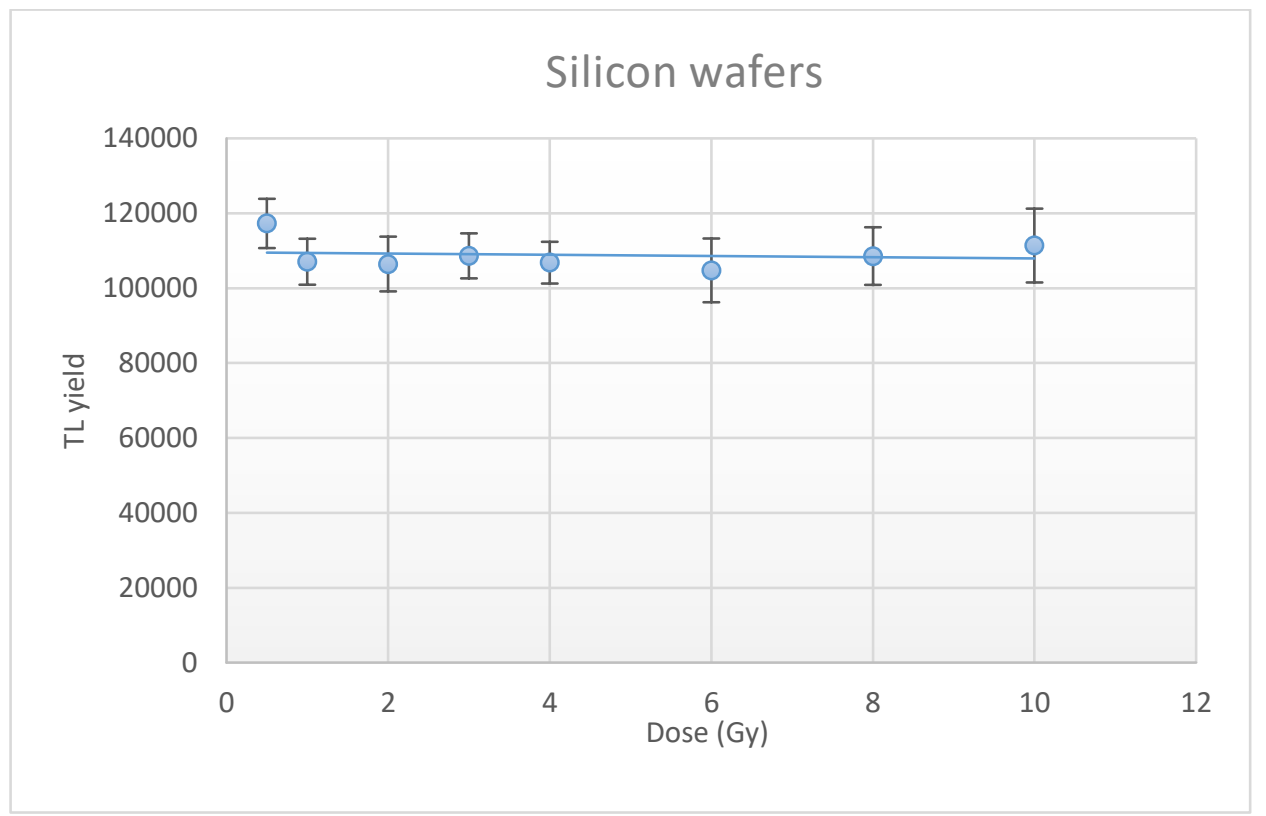

Figure 5. Results for the silica wafer samples, irradiated at eight dose points, each dose point being represented as the mean of three wafer samples. The lack of TL response is seen to be direct effect of the extremely low defect density and negligible doping in the medium. The minimal response seen at low dose is suggested to relate to surface oxidation, giving rise to minimal trapping levels, saturated at low dose. 


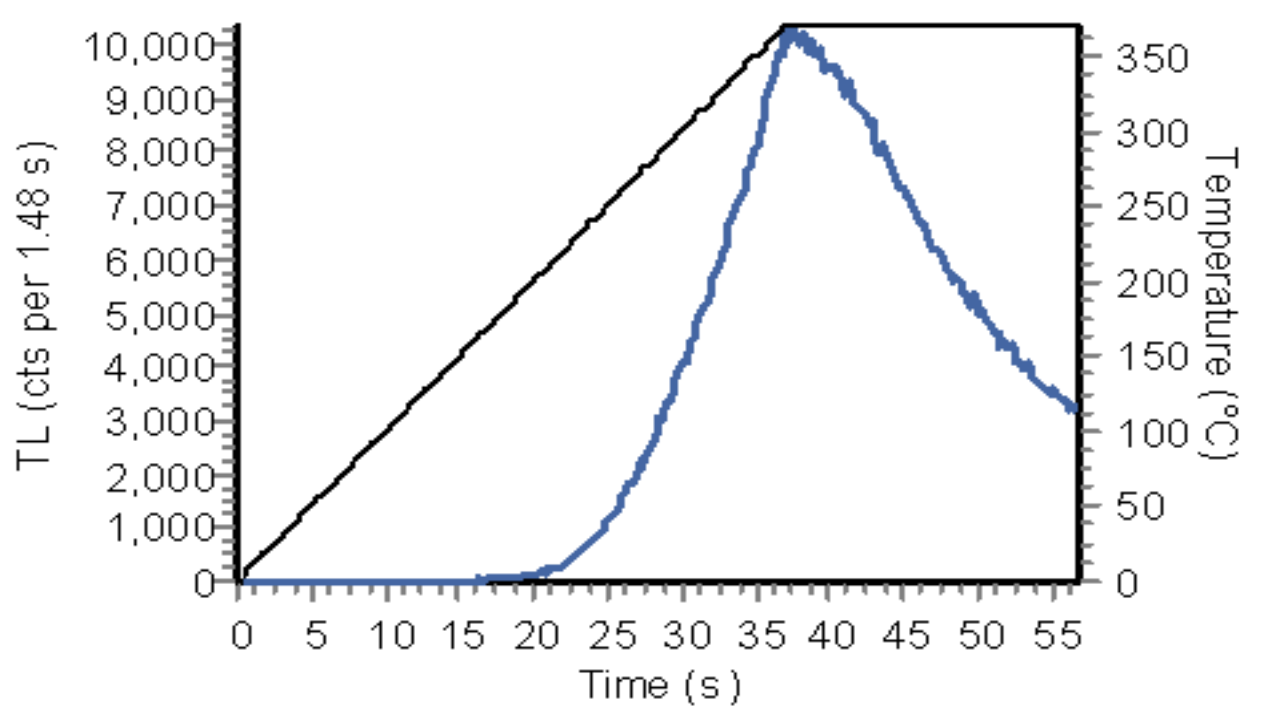

Figure 6. Glow curve of borosilicate glass slides irradiated to a dose of $10 \mathrm{~Gy}$ at $250 \mathrm{kVp}$

\section{Reproducibility for Borosilicate Glass slides}

Using the Gulmay unit, reproducibility was investigated for 56 randomly selected glass slide samples, for readout being divided into two irradiated groups (noting the limit on accommodation of large sample numbers in the TL reader). The first group of 28 were exposed to a uniform dose of $1 \mathrm{~Gy}$ at $250 \mathrm{kVp}$, field size $(20 \mathrm{x} 20 \mathrm{~cm})$. The combined standard deviation of the entire TLD process for the 56 samples was chosen to provide for an allowance of $\pm 5 \%$, discarding outlier samples, noting that radiotherapy dosimetry seeks to remain well within $\pm 5 \%$,. A second exercise exposed the remaining group of slides to a dose of $2 \mathrm{~Gy}$, the same field size being used. As can be seen in Figs. 7 and 8, shown for 28 glass slide samples in each case, there is strong evidence of a high degree of reproducibility with relatively few needing to be discarded from further study.

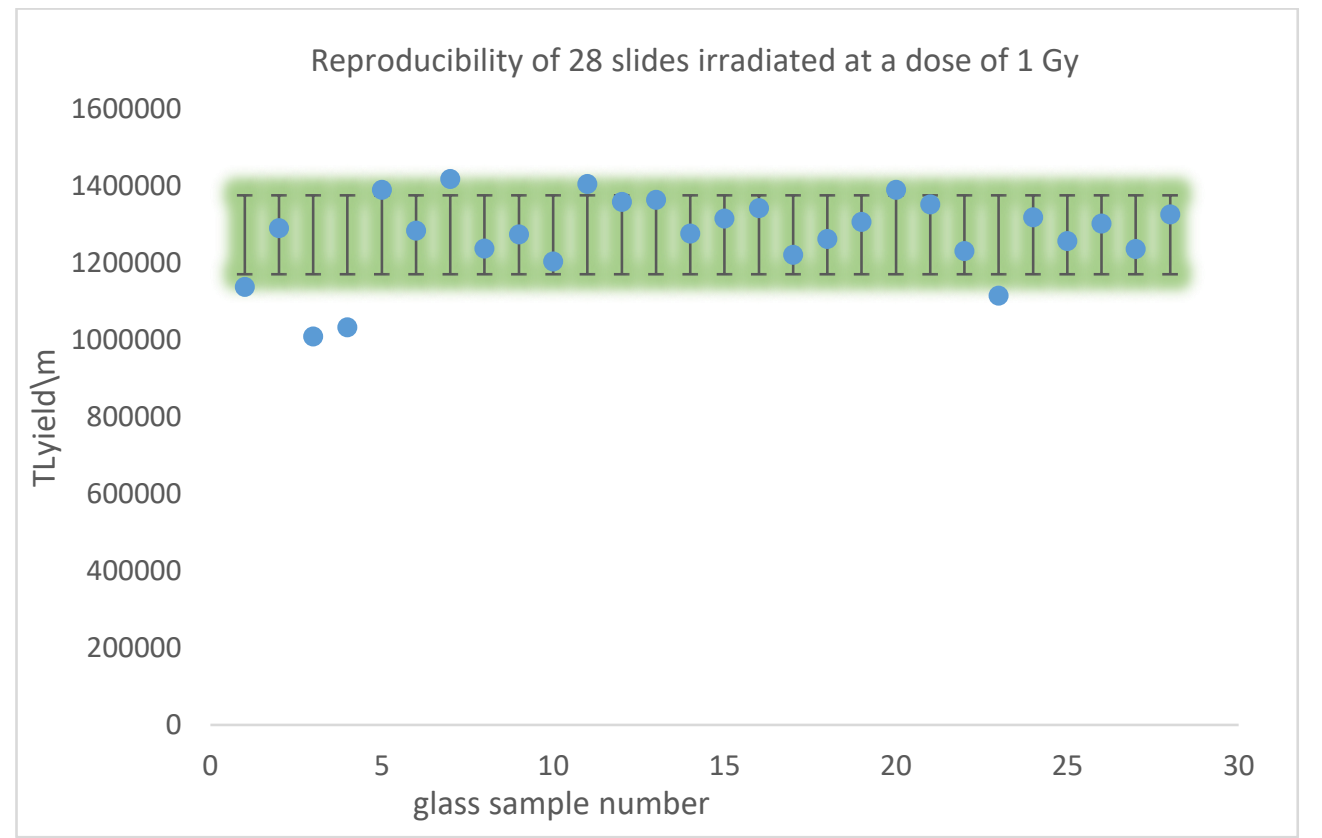

Figure 7. Reproducibility of the first group of glass slide samples (28 in all); outliers are easily screened out in such an exercise 


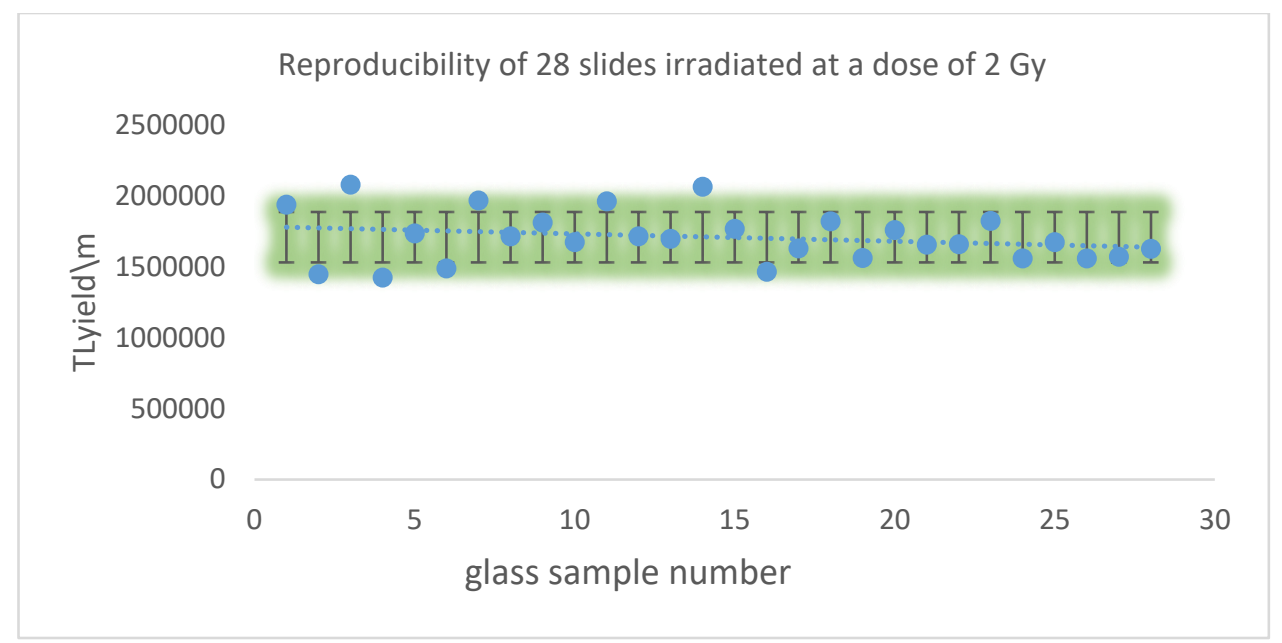

Figure 8. Reproducibility of the second group of glass slide samples (28 in all); outliers are easily screened out in such an exercise.

\section{Study of Linearity for MV photons and MeV electrons:}

Ideally, a good dosimeter should exhibit linear response over a wide range of irradiation doses, borosilicate glass for example exhibiting such dynamic range in use of the orthovoltage x-ray unit. Similarly, for 6 and 10 MV photon beams, obtained using a linac and covering doses ranging from 10 cGy to $2000 \mathrm{cGy}$, the response of the borosilicate glass slides was also found to be linear (Figs. 9). Of particular interest in terms of the borosilicate makeup, specifically the boron content, is the associated appreciable neutron cross-sections of the latter. It is posited that the additional response observed at $10 \mathrm{MV}$ over that at $6 \mathrm{MV}$ is partly a result of photo-neutron production, a situation that arises around the $10 \mathrm{MV}$ threshold level, a matter which is beyond present scope but which is expected to form the basis of further investigations by this group.

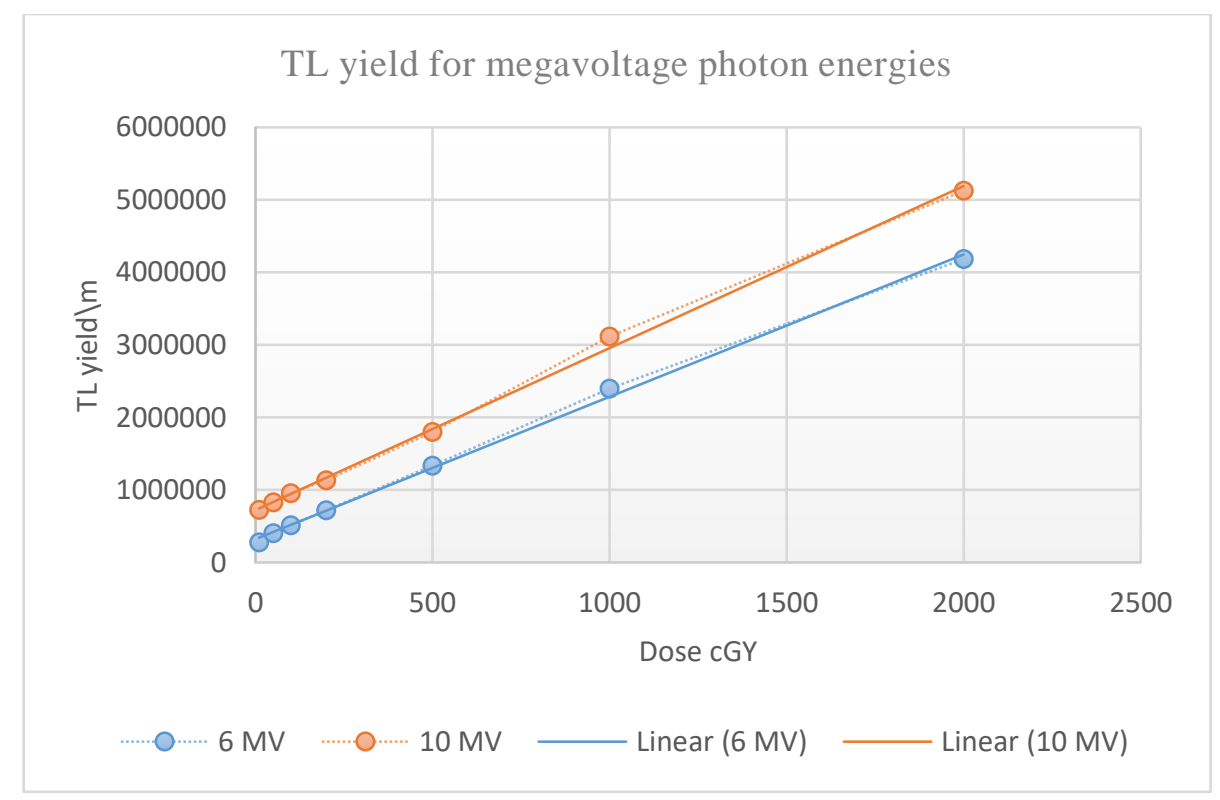

Figure 9. Linearity of TL response of the borosilicate glass slides of the same nominal thickness, for doses ranging from 10 cGy to 2000 cGy, delivered by megavoltage photons. 
In regard to electron irradiations, extending from $6 \mathrm{MeV}$ through to $16 \mathrm{MeV}$, in respect of the lower two energies (6 and $9 \mathrm{MeV}$ ) closely linear response is noted (Fig. 10). Conversely, for the two greater irradiation energies (12 and $16 \mathrm{MeV}$ ) there is indication of possible approach towards detection saturation, potentially as a result of LET dependence. Alternatively, a further consideration is that the behaviour may result from potential sample thickness-dependent bremsstrahlung loss, a matter currently under investigation.

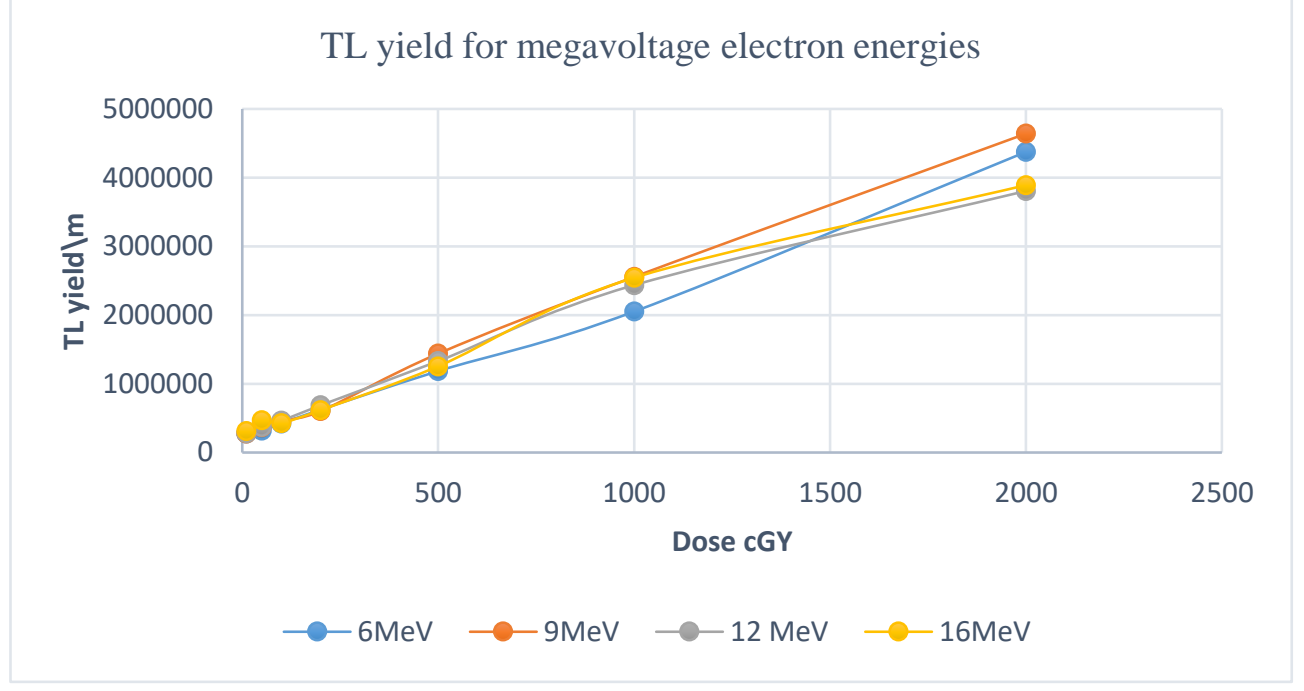

Figure 10. TL response of the borosilicate glass slides for doses ranging from 10 cGy to 2000 cGy for megavoltage electron energies. The points relate to the mean value of 5 individual samples

\section{Study of energy dependence using photon irradiations:}

Using the borosilicate glass slide samples, investigation has been made of beam energy dependence for the range of photon beams available during the course of present studies, $80 \mathrm{kVp}$ through to $10 \mathrm{MV}$. Fig. 11 shows the resulting comparative TL yield, normalized to that obtained at $6 \mathrm{MV}$. Unsurprising is the photoelectric dependence obtained at the lower beam values, obtained through use of the Gulmay x-ray machine, reflecting an effective atomic number of the glass media of some 11.4 (dominated by the fractional $\mathrm{Si}$ and $\mathrm{O}_{2}$ content of the medium, as shown in Table 1). The substantial increase in response at $10 \mathrm{MV}$ compared to that at $6 \mathrm{MV}$ is in part a result of an increase in pair production probability while the boron content of the glass may contribute to a neutron production contribution at a beam energy just above the photoneutron production threshold energy.

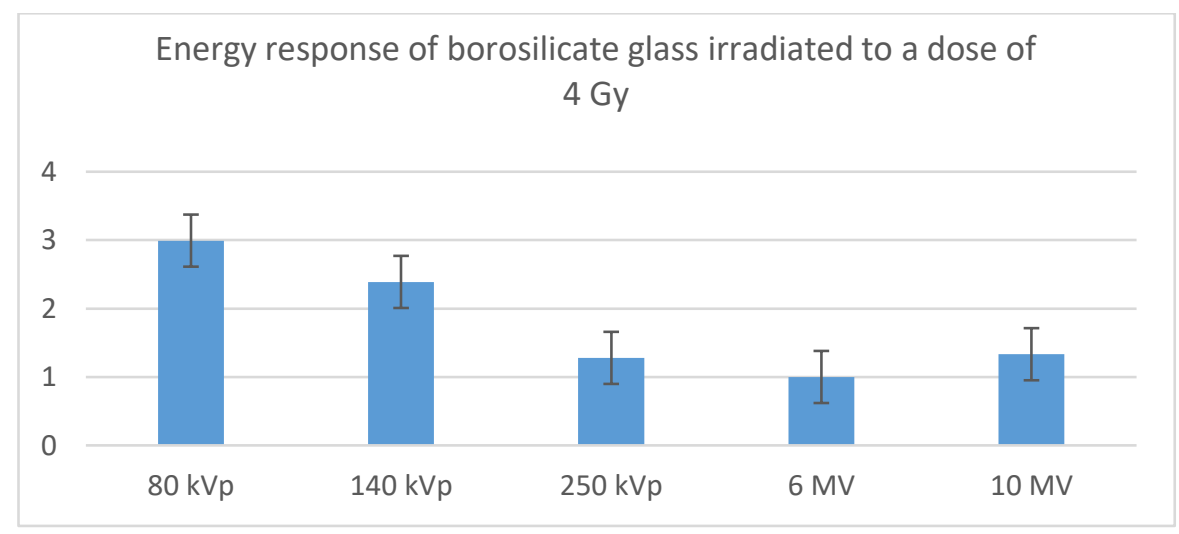

Figure 11. TL response of borosilicate glass slides irradiated to a dose of $4 \mathrm{~Gy}$ with $\mathrm{kV} X$-rays $(80,140$ and $250 \mathrm{kVp})$ and megavoltage photons ( 6 and $10 \mathrm{MV}$, all normalised to the response at $6 \mathrm{MV}$. 


\section{Discussion and Conclusions:}

Exploratory results have been presented for two potential low-cost glass dosimeters, evaluated for potential use in radiotherapy dosimetry. Investigated in terms of their thermoluminescence yield, the dose response results have been contrasted against that from a defect free/dopant free glass medium in which an effective null response is obtained. The borosilicate microscope slide medium is seen to have excellent potential for radiotherapy dosimetry utilisation, from orthovoltage x-ray levels through to megavoltage. A substantial TL yield is also seen for the low density glass fibre filters, measured in terms of photon response at $250 \mathrm{kVp}$ for a typical range of radiotherapy doses. The same is not true of the silica wafers, the low TL yield being due to the use of high purity silica, devoid of defects. Interest is developing in the potential dosimetric utility of the borosilicate glass and its neutron response, with initial indications resulting from observations made at $10 \mathrm{MV}$ over that at $6 \mathrm{MV}$, posited to be partly a result of photo-neutron production, a situation that arises around the $10 \mathrm{MV}$ threshold level.

\section{References:}

Allahverdi, M., Nisbet, A. and Thwaites, D. (1999). An evaluation of epoxy resin phantom materials for megavoltage photon dosimetry. Physics in Medicine and Biology, 44(5), pp.1125-1132.

Bradley, D.A., Mahdiraji, G.A., Ghomeishi, M., Dermosesian, E., Adikan, F.R.M., Abdul Rashid, H.A., Maah, M.J. (2015). Enhancing the radiation dose detection sensitivity of optical fibres. Applied Radiation and Isotopes 100, 4349.

Bradley, Jafari, S.M., Siti Shafiqah A.S., Tamcheck N., Shutt, A., Z.Siti Rozaila, Z., Abdul Sani, S.F., Siti Norbaini Sabtu, Abdulaziz Alanazi, Amouzad Mahdiraji, G., Abdul Rashid, H.A., Maah, M.J. (2016). Latest developments in silica-based thermoluminescence spectrometry and dosimetry (Applied Radiation and Isotopes 117, 128-134.

Knoll, G.F., 2000. Radiation Detection and Measurement, John Wiley \& Sons. Inc., New York, 2000.

Laboratory Resource. (2019). Borosilicate Glass. [online] Available at:

https://laboratoryresource.com/?navaction=getitem\&id=225 [Accessed 29 Jul. 2019].

Ley, K., Jafari S.M., Lohstroh A., Shenton-Taylor, C., Bradley, D.A. Thermoluminescent response of beta-irradiated silica beads, (2019). Radiation Physics and Chemistry, 154, 32-37.

Liuzzi, R., Savino F., D’Avino, V., Pugliese, M., and Cella, L. (2015). Evaluation of LiF:Mg,Ti (TLD-100) for Intraoperative Electron Radiation Therapy Quality Assurance. PLOS One. https://doi.org/10.1371/journal.pone.0139287

Physics.nist.gov. (2019). Composition of Pyrex Glass. [online] Available at: https://physics.nist.gov/cgibin/Star/compos.pl?matno=169 [Accessed 29 Jul. 2019].

Institute of Physics and Engineering in Medicine (IPEM) Report 81. (1999), Chapter 1.

Microchemicals.com. (2019). microchemicals: Wafer, Si-Wafer, Silicon, Offer, Request, Production. [online] Available at: https://www.microchemicals.com/products/wafers [Accessed 29 Jul. 2019].

Turner, J.E., Atoms, radiation, and radiation protection. Wiley-VCH. 2008.

Yaakob N.H. , Wagiran H. , Hossain M.I. , Ramli A.T. , Bradley D.A. , Ali H. (2011). Low-dose photon irradiation response of Ge and Al-doped SiO2 optical fibres. Applied Radiation and Isotopes. 69, 1189-1192. 